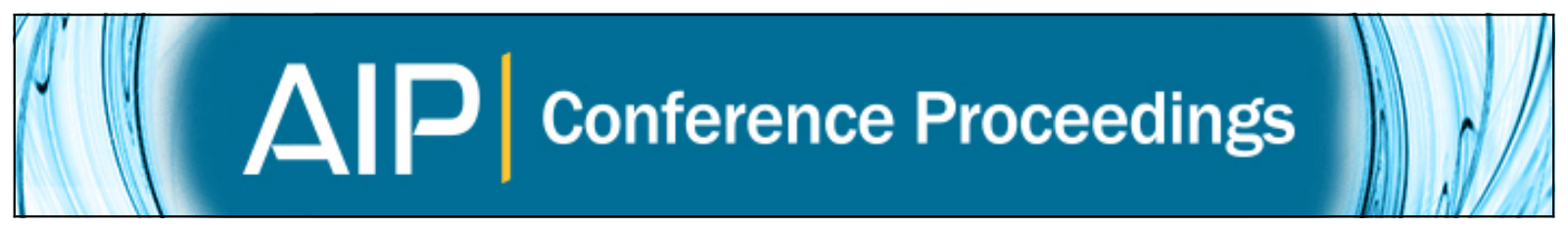

\title{
A novel achromatic Fresnel lens for high concentrating photovoltaic systems
}

Guido Vallerotto, Stephen Askins, Marta Victoria, Ignacio Antón, and Gabriel Sala

Citation: AIP Conference Proceedings 1766, 050007 (2016); doi: 10.1063/1.4962089

View online: http://dx.doi.org/10.1063/1.4962089

View Table of Contents: http://scitation.aip.org/content/aip/proceeding/aipcp/1766?ver=pdfcov

Published by the AIP Publishing

\section{Articles you may be interested in}

Design and characterization of a curved linear Fresnel lens concentrating photovoltaic and thermal system AIP Conf. Proc. 1616, 173 (2014); 10.1063/1.4897054

Estimation of the Influence of Fresnel Lens Temperature on Energy Generation of a Concentrator Photovoltaic System

AIP Conf. Proc. 1407, 97 (2011); 10.1063/1.3658303

High Performance Concentrating Photovoltaic Module Designs Employing Reflective Lens Optics AIP Conf. Proc. 1407, 70 (2011); 10.1063/1.3658297

Optical Analysis of Fresnel lens for concentrating PV system

AIP Conf. Proc. 1207, 244 (2010); 10.1063/1.3366373

An achromatic retarding mode for the Menzinger lens system

Rev. Sci. Instrum. 62, 2044 (1991); 10.1063/1.1142365 


\title{
A Novel Achromatic Fresnel Lens for High Concentrating Photovoltaic Systems
}

\author{
Guido Vallerotto $^{\text {a)}}$, Stephen Askins, Marta Victoria, Ignacio Antón, Gabriel Sala \\ Instituto de Energía Solar, Universidad Politécnica de Madrid, Ciudad Universitaria 28040, Madrid, Spain \\ ${ }^{a)}$ Guido Vallerotto: guido.vallerotto@ies-def.upm.es
}

\begin{abstract}
In this paper we present a novel manufacturing method to produce achromatic Fresnel lenses for photovoltaic application. These achromatic lenses are capable of reaching a concentration factor three times higher than that attained by a conventional Silicone-on-Glass (SOG) Fresnel lens. The manufacturing method presented to fabricate the achromatic lens, which we refer to as Achromatic Doublet on Glass (ADG) Fresnel lens, is simple, cost-effective and highly scalable. A comprehensive ray-tracing analysis and its comparison with experimental results is presented in this work.
\end{abstract}

\section{INTRODUCTION}

High concentrating photovoltaic (HCPV) systems have the capacity to reduce the cost of solar-based electricity because they can take advantage of the rapid increase in the efficiency of advanced multijunction (MJ) solar cells. This type of cell has attained the highest recorded efficiency, reaching values higher than $46 \%$ [1]. Currently, most of the commercially available HCPV systems are based on Silicone on Glass (SOG) hybrid Fresnel lenses [2]. The injection-molded SOG process features a rigid glass substrate to which a Fresnel lens structure composed of optical silicone rubber is directly molded.

Fresnel lenses, like any refractive optical system, suffer from the phenomenon of chromatic aberration. Chromatic aberration is due to the materials' refractive index variation as a function of the incident light wavelength. Because of the chromatic aberration, in a positive (converging) lens, light at given short wavelength (which we will refer to as "blue" light) will intersect with the optical axis at a point closer to the lens than light at some longer wavelength ("red" light), as shown in Fig. 1a.

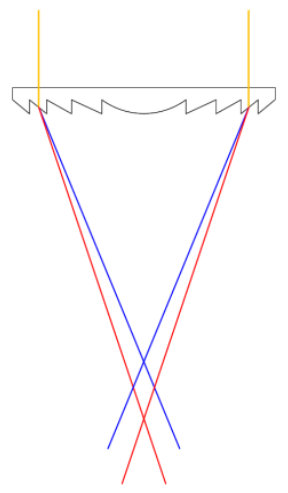

(a)

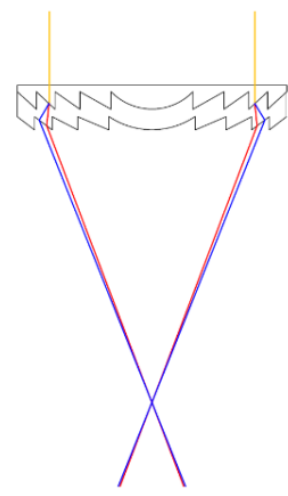

(b)

FIGURE 1. a) Schematic representation of chromatic aberration phenomena in a classic Silicon on Glass (SOG) Fresnel lens. b) Schematic representation of the chromatic aberration correction obtained using the proposed Achromatic Doublet on Glass (ADG) Fresnel lens.

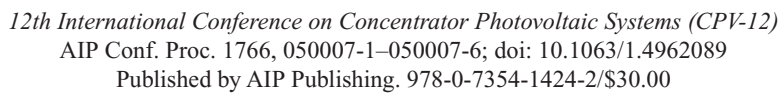


Figure $1 \mathrm{~b}$ represents an optical refractive system composed of two Fresnel lenses made from two materials having distinct dispersion. Such a lens is able to correct for chromatic aberration, forcing blue and red light rays to intersect the optical axis at the same point. This kind of lens is named "Achromatic doublet" and it is known since $18^{\text {th }}$ century when Joseph Von Fraunhofer carried out a comprehensive research studying their design method and manufacturing process [3]. Achromatic doublets are usually manufactured out of two types of glasses using expensive processes, and their cost is incompatible with the photovoltaic industry. In 2011 Languy and co-workers [4] proposed an achromatic doublet for CPV composed of two plastics: poly(methyl methacrylate) (PMMA) and polycarbonate (PC). They did a comparative analysis on different configurations and their advantages but they did not describe a manufacturing process that can be scalable at high production level at low cost.

In our work we have gone one step further, proposing a simple, reliable, and cost-effective manufacturing method to produce achromatic lenses for HCPV applications. As shown in Fig. 2, our design, which we refer to as Achromatic Doublet on Glass (ADG) Fresnel lens, includes an elastomeric material with low dispersion, a preformed plastic lens with high dispersion, and a rigid substrate made of glass ${ }^{1}$.

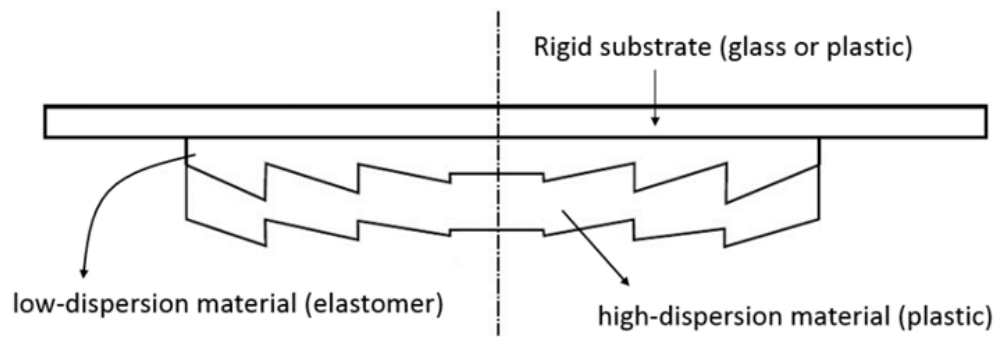

FIGURE 2. Schematic representation of the materials comprising the ADG lens. The plastic operates as a mold shaping the geometry of the elastomeric lens; the elastomer, in turn, acts as a glue coupling plastic lens and the rigid substrate.

First, a plastic "bi-Fresnel" lens (a thin lens with Fresnel grooves on both surfaces) is manufactured using standard injection molding, compression molding or hot embossing processes, both of which are inexpensive, reliable and commonly used in high volume industry. Then, a lamination process is used to join the bi-Fresnel lens to the rigid transparent substrate, with the elastomeric material serving both as an adhesive while simultaneously being formed into the low dispersion lens of doublet. The lamination step could be similar to that used in the manufacture of flat-plate silicon solar panels. Since both mentioned processes (plastic injection or hot embossing, and lamination) are inexpensive and highly reliable we expect the final overall manufacturing cost of ADG Fresnel lenses to be similar to the fabrication cost of classic SOG Fresnel lenses. Hence, the increase in concentration thanks to the achromaticity is attained practically at no extra cost.

\section{DESIGN OF THE ACHROMATIC FRESNEL LENS}

The optical design is performed by imposing an "achromaticity condition" for two wavelengths, that is designing the Fresnel facets in such a way that blue light and red light have exactly the same focal distance (see Fig. 1b). The general design method for achromatic doublets is well documented [5], while a detailed disclosure relating to our specific design will be presented shortly [6].

\section{COMPARATIVE ANALYSIS BASED ON RAY-TRACING SIMULATIONS}

A comprehensive set of ray-tracing simulations was performed in order to predict and analyze the performance of the ADG Fresnel lens previously described. Details about parameters and criteria as well as an extensive disclosure of obtained results will be available shortly [6].

Main results that is worth to summarize again here are the maximum attainable concentration, the curve representing the acceptance angle vs. the geometric concentration, and the size of the generated light spot. The maximum attainable concentration for the ADG Fresnel lens has been found to be almost three times higher than for

\footnotetext{
${ }^{1}$ We note that a transparent plastic substrate, for instance poly(methyl methacrylate) (PMMA), could be used; however we assume that glass is preferred for all its properties such as flatness, UV-filtering, resistance to degradation, etc.
} 
an equivalent SOG Fresnel lens. Compared to a classic SOG Fresnel lens, the ADG lens allows increasing the concentration from approximately 250X to 700X. The concentration-acceptance angle (CAP) is larger for the ADG lens than for the SOG lens with the same optical aperture and focal distance. In other words, the curve representing acceptance angle vs. geometric concentration for any considered point is higher for the ADG Fresnel lens than for the classic SOG meaning that we can choose either to increase the concentration maintaining the same acceptance angle or to keep the same concentration and use the ADG lens to widen the acceptance angle, thus increasing the tolerance.

\section{Size of the Irradiance Spot}

In this section we discuss the size of the irradiance spot and the achromaticity behavior of ADG Fresnel lens. In the simulations described in this section top subcell receiver (receiving light whose wavelength is lower than 660 $\mathrm{nm}$ ) and middle subcell receiver (receiving light whose wavelength is comprised between 650 and $900 \mathrm{~nm}$ ) have been analyzed separately. In this way it was possible to analyze ADG concentrating capability for blue light, converted by the top subcell, and for red light, converted by the middle subcell. Bottom subcell has not been considered because refractive index variation beyond $900 \mathrm{~nm}$ are minor, as a consequence the lens behavior with light having wavelengths longer than $900 \mathrm{~nm}$ will be quite similar to its behavior between 650 and $900 \mathrm{~nm}$.

Receivers (both top and middle) position varies from five $\mathrm{mm}$ closer to the lens than the optimal position to five $\mathrm{mm}$ further from the optimal position. Optimal position is the focal distance at which the spot size is minimized.

Measuring the size of irradiance spot in each position for both receivers allowed characterizing the spot diameter variation as a function of the receivers' position. Blue curves refer to blue light while red dashed ones to red light.

\section{SOG Fresnel lens}

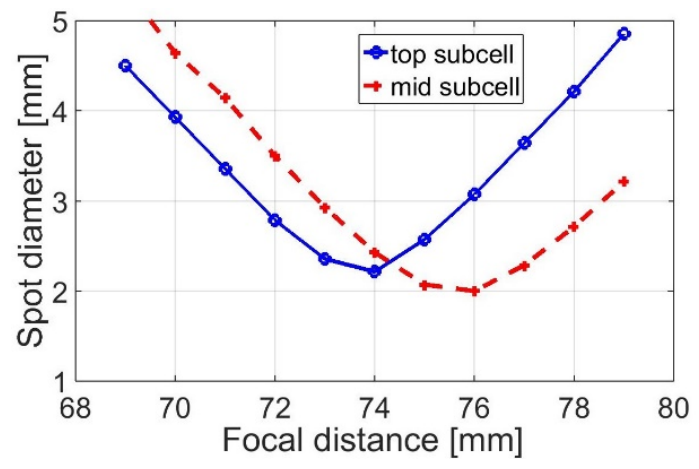

(a)

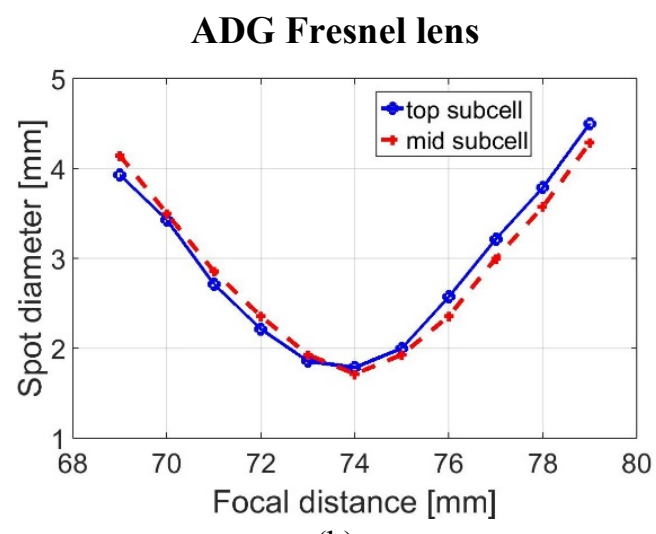

(b)

FIGURE 3. Spot diameter predicted by ray-tracing as a function of the receiver displacement from the optimal focal distance (minimum spot). Spot diameter is defined as that including 99\% of the energy. Red dashed lines represent spot diameters for longer wavelengths (those typically converted by middle subcell in MJ solar cells, i.e.: 650-900nm) and blue continuous lines represent spot diameters for shorter wavelengths (those typically converted by top subcell, i.e.: $350-650 \mathrm{~nm}$ ).

For the classic SOG Fresnel lens, the minimum of the two curves are displaced by about $2 \mathrm{~mm}$ as shown in Fig. 3a. This is due to the chromatic aberration: since refractive index for short wavelength is higher, the focal point for blue light is closer to the lens than the one for the red light (remember Fig. 1) and so the minimum spot for blue light is displaced to the left (toward the lens) and the minimum spot for red light displaced to the right (toward infinite). Notice that to collect all the light useful for the MJ solar cell it should be placed at the position where the maximum of the two spots (corresponding to top and middle subcells) is minimized), Conversely, in Fig. 3b we see that, for the ADG Fresnel lens, the minimum spot for blue light corresponds exactly with the minimum spot for red light, demonstrating its achromaticity.

\section{MANUFACTURING PROCESS}

The manufacturing process proposed in this work is essentially divided in two steps: the plastic injection of the "bi-Fresnel" lens and the lamination of this plastic part to a glass substrate with an optical encapsulant. Common thermoplastic forming techniques, such as plastic injection molding, compression injection molding, and hot 
embossing could be used to obtain de "bi-Fresnel" lens. These techniques are commonly used in many different fields. It should be noted that manufacturing tolerances (i.e. tip rounding and draft angles) are slightly different for plastic parts versus silicone molding. For example silicone injection allows a minimum tip rounding value of three microns while PC of about five microns.

\section{Lamination Process}

This step has been carried out in our labs at the IES-UPM using a built-in prototype laminator shown in Fig. $4 \mathrm{a}$.

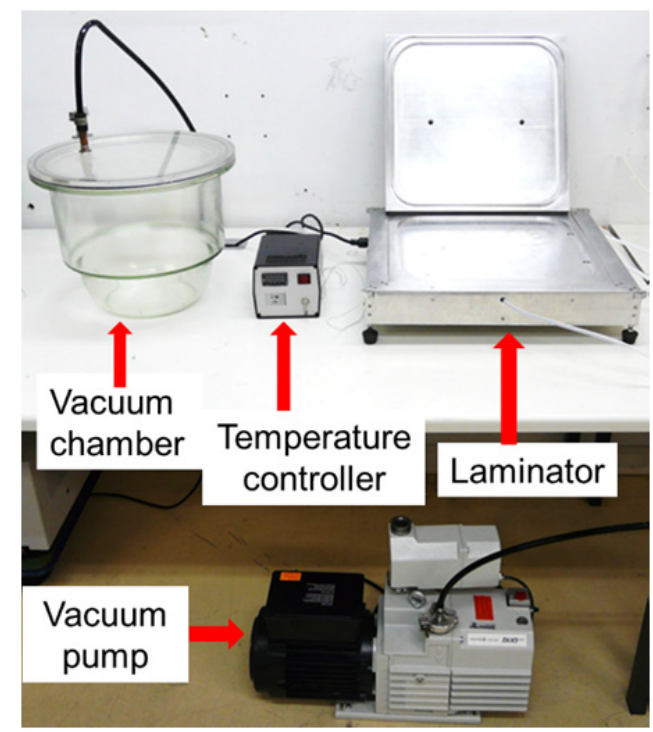

(a)

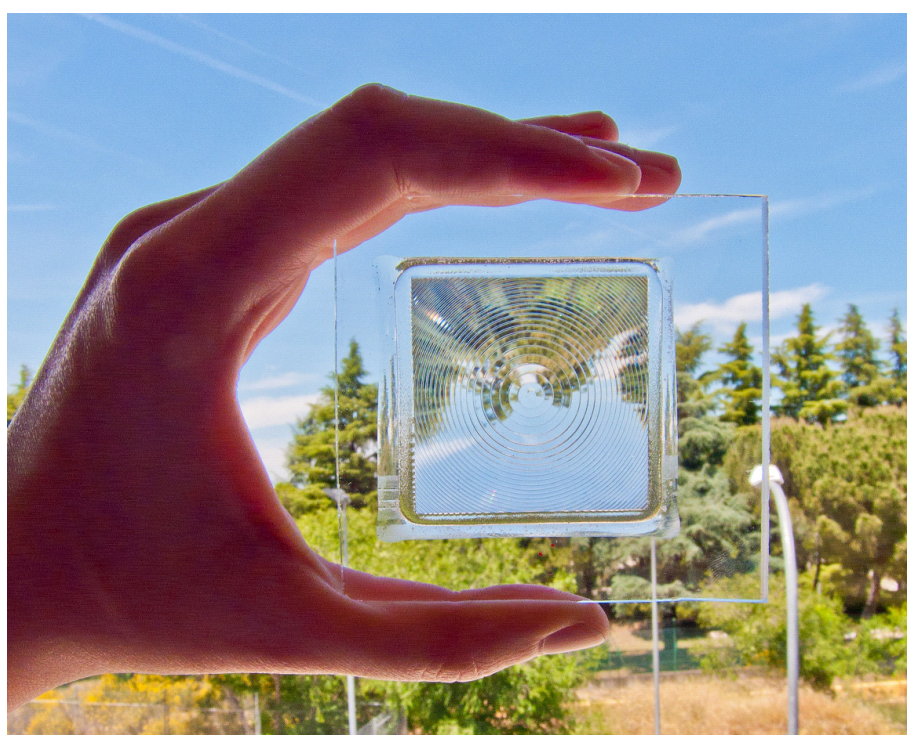

(b)

FIGURE 4. a) Picture of the prototype laminator built at the IES-UPM and used to laminate the first ADG Fresnel lens prototype. b) The ADG prototype was laminated at the IES-UPM. No defect can be observed by the naked eye.

The lamination process is divided in three steps: degasification, lamination, and curing phase. An optimization of manufacturing parameters such as the duration of each step and temperature and pressure at which they are carried out has been realized. Using optimized parameters we could easily manufacture the prototype shown in Fig. 4b which has a very good transparency and it is free of air bubbles.

\section{EXPERIMENTAL RESULTS}

In this section experimental results obtained for the first ADG Fresnel lens prototype and for an equivalent SOG Fresnel lens provided by Fraunhofer-ISE. are presented. Both lenses have an optical aperture of $40 \times 40 \mathrm{~mm}$. The most important results concern the size of the irradiance spot and the achromaticity behavior of the ADG.

\section{Experimental Setup}

Figure 5 shows a functional scheme of the laboratory setup used to carry out experimental testing (Fig. $5 \mathrm{a}$ ) and a picture of the same system (Fig. 5b).

The setup comprises:

- A collimated light source having an angular aperture equal to $\pm 0.27^{\circ}$ composed of a lamp within an integrating sphere and a large area parabolic mirror used to collimate the light beam proceeding from the source.

- The lens sample (ADG or SOG) with a moving support through which it is possible to adjust the distance between lens and camera.

- Low pass or high pass filters with a cutoff of approximately $670 \mathrm{~nm}$.

- A CCD sensor. 


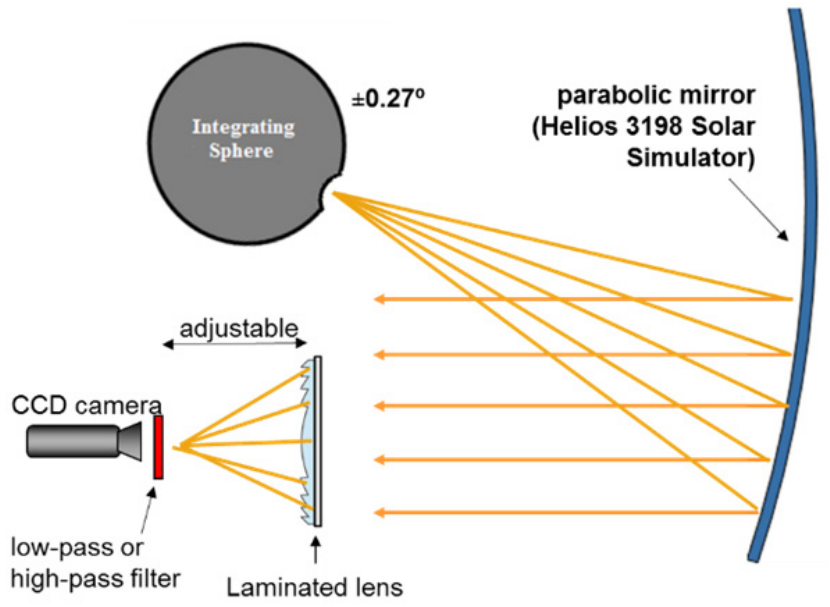

(a)

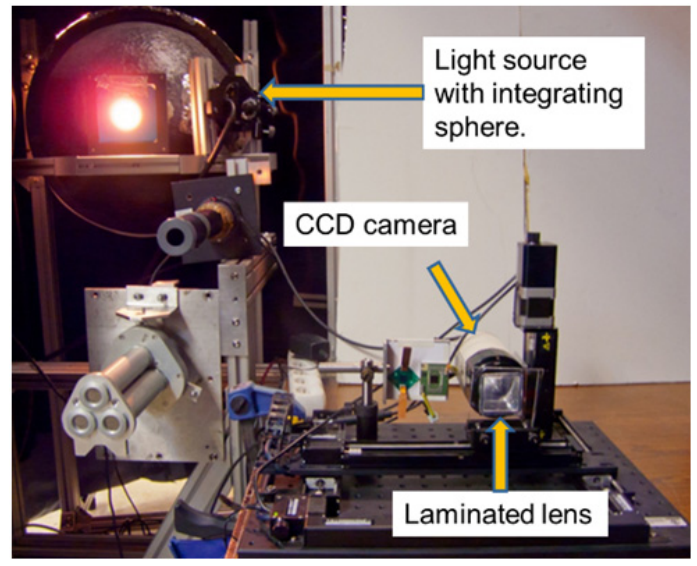

(b)

FIGURE 5. a) Scheme of the experimental setup used to carry out experimental testing. b) Picture of the experimental setup and its components (light source with integrating sphere, lens sample, and CCD camera. Parabolic mirror and filters are not visible in this picture).

The CCD sensor was placed near the focus of the sample lens, and by processing the resulting image it is possible to get the diameter of the spot. Low- and high-pass diffractive filters with appropriate cut-off frequencies were used to selectively image only light converted by top or middle subcells depending on which filter has been used. By moving the lens support with respect to the CCD array, it is possible to move the lens closer or further from the camera. In this way we were able to locate the optimal position (minimum spot diameter) for both tested lenses and for both subcells.

\section{Results and Discussion}

The evolution of the spot diameter as a function of the lens position with respect to the camera for both lenses and subcells is shown in Fig. 6. This figure is similar to Fig. 3 but represents experimental data instead of ray-tracing simulation results.

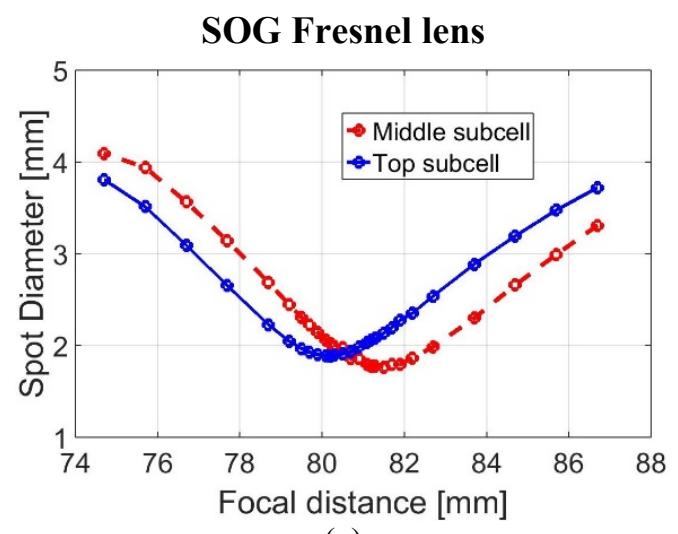

(a)

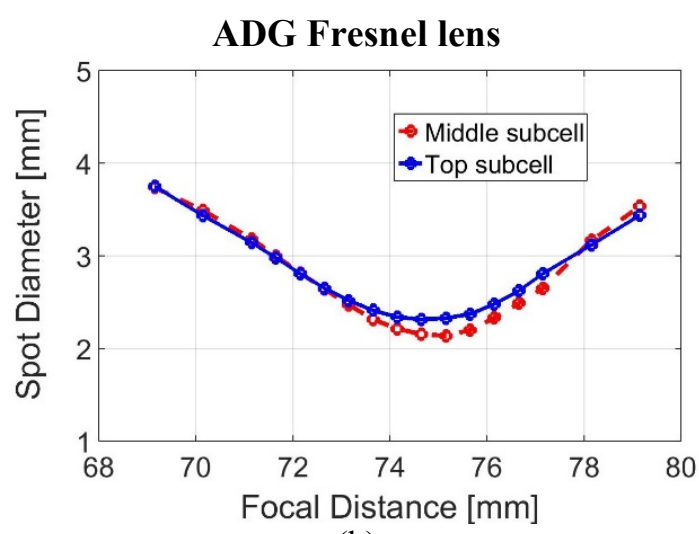

(b)

FIGURE 6. Measured spot diameter as a function of the receiver displacement from the optimal focal distance (minimum spot). Spot diameter is defined as that including $95 \%$ of the energy. Red dashed lines represent spot diameters for longer wavelengths (those typically converted by middle subcell in MJ solar cells, i.e.:650-900nm) and blue continuous lines represent spot diameters for shorter wavelengths (those typically converted by top subcell, i.e.: $350-650 \mathrm{~nm}$ ). 
First, by comparing Fig. 6 with Fig. 3, we can see that the achromatic behavior of the ADG lens predicted by ray tracing simulations has been verified by experimental results. In fact, in Fig. 6 it is possible to appreciate the same displacement between minimum points of blue and red curves for the SOG lens while, as expected, minimum for blue and red curves for the ADG lens coincides. This confirms correctness and reliability of our design method and manufacturing process.

However we observe that ray tracing predicts that the minimum spot size produced by ADG lenses will be smaller than those of SOG, the experimental results showed the opposite. We believe this is caused by two factors:

1. Both lenses under study, SOG and ADG, have the same optical aperture, $40 \times 40 \mathrm{~mm}$. On the one hand, the lenses analyzed by ray-tracing have the same focal distance (approximately $74 \mathrm{~mm}$ ). Hence, the f-number considering the lens diagonal is 1.31 for both cases. On the other hand, the experimental results show that the focal distance for the SOG lens is slightly longer than that of the ADG lens. Based on that, the f-number experimentally measured for the SOG would be 1.41. For classic Fresnel lenses the relation between attainable concentration and f-number has a maximum for an f-number value of about two [7] and decrease for lower values. The longer focal distance measured for the SOG lens may be casting a reduced size spot.

2. The reference SOG designed for ray-tracing simulation, as well as the present ADG lens design, have flat facets in order to ease design and potential manufacturing process. The SOG Fresnel lens provided by Fraunhofer-ISE features curved facets resulting in an improved concentrating power. By incorporating curved facets into our design we should be able to reduce our spot size further.

\section{CONCLUSIONS}

A novel achromatic lens concept applicable to HCPV and its manufacturing method have been presented. The proposed manufacturing process is at the same time simple, cost-effective, and reliable. The primary manufacturing steps include the manufacturing of a bi-Fresnel lens (by injection molding, compression molding or hot embossing) and its lamination with an optical elastomer to a glass substrate. The ease with which we were able to prototype the lens together with low cost and reliability of manufacturing process suggest that the method will be easily scalable to volume production and its overall cost will be comparable to the cost of the manufacturing process currently used to fabricate classic SOG Fresnel lenses.

Ray-tracing simulations suggest that in the same optical system, substituting a classic SOG Fresnel lens with the ADG Fresnel lens, it is possible to increase the maximum concentration from $250 \mathrm{X}$ to $700 \mathrm{X}$. Reliability of the proposed manufacturing method and achromaticity behavior of the fabricated ADG lens prototype have been verified via both simulation and experimental measurements.

Results of this work are very promising for the future development of the ADG Fresnel lens; next steps in our research include the improvement of the design (e.g. including curved facets) and of the manufacturing method in order to increase both optical efficiency and maximum attainable concentration.

\section{ACKNOWLEDGMENTS}

This work has been partially supported by the Spanish Ministry of Economy and Competitiveness under the Acromalens project (ENE2013-45229-P) and it has received funding from the European Union's Horizon 2020 research and innovation program within the project CPVMatch under grant agreement No 640873.

\section{REFERENCES}

1. M.A. Green, K. Emery, Y. Hishikawa, W. Warta, and E.D. Dunlop, Prog. Photovolt. Res. Appl. 24, 3 (2016).

2. $\quad$ E. Lorenzo and G. Sala, in Sun II (1979), pp. 536-539.

3. D. Lemke, Investig. Cienc. 472, 70 (n.d.).

4. F. Languy, K. Fleury, C. Lenaerts, J. Loicq, D. Regaert, T. Thibert, and S. Habraken, Opt. Express 19 Suppl 3, A280 (2011).

5. $\quad$ E. Hecht, Optics, Third Edition (Addison Wesley Longman, Inc., 1998).

6. G. Vallerotto, M. Victoria, S. Askins, R. Herrero, C. Domínguez, I. Antón, and G. Sala, Opt. Express (Submitted)

7. M. Victoria, New Concepts and Techniques for the Development of High-Efficiency Concentrating Photovoltaic Modules, PhD, E.T.S.I. Telecomunicación (UPM), 2014. 